\title{
Protease Activity in Giardia duodenalis Trophozoites of Axenic Strains Isolated from Symptomatic and Asymptomatic Patients
}

\author{
Semíramis Guimarães $/{ }^{+}$, Maria Inês Terra Leme Sogayar, Marcello Fabiano Franco*
}

Departamento de Parasitologia, Instituto de Biociências, Universidade Estadual Paulista, Campus de Botucatu, 18618-000 Botucatu, SP, Brasil *Departamento de Patologia, Escola Paulista de Medicina, Universidade Federal de São Paulo, São Paulo, SP, Brasil

We have examined by gelatin-SDS-PAGE the protease activity in cell lysates of Giardia duodenalis trophozoites of two axenic strains isolated in Brazil from a symptomatic patient (BTU-11) and an asymptomatic carrier (BTU-10), and the reference strain Portland 1 (P1). The proteolysis band patterns showed differences among strains isolated from asymptomatic and symptomatic individuals. The lysate of the strain BTU-10, showed only five hydrolysis bands, while a greater number of bands (10-11 bands) was seen in strains BTU-11 and P1. The protease activity in all lysates was inhibited by cysteine (E-64 and iodoacetamide) and serine proteases (TPCK and TLCK) inhibitors, but not by PMSF and EDTA. In general, the results revealed protease activities in G. duodenalis trophozoites of Brazilian axenic strains and the predominance of cysteine proteinases. It should be stressed the inter-strain difference in hydrolysis band patterns observed between strains isolated from symptomatic patients and the strain obtained from an asymptomatic carrier.

Key words: Giardia duodenalis - axenic strain - trophozoites - protease activity

Giardia duodenalis (syn. G. lamblia, G. intestinalis) is a flagellated protozoan found in the small intestine of man and other mammals. Giardiasis is currently considered to be an important public health problem in developing countries, where it is one of the most common causes of diarrhea among children.

Recent advances in the understanding of the biochemistry and molecular biology of protozoa have permitted the study of molecules that directly participate in the parasites life cycle or in the pathogenicity (North et al. 1990). Many important parasite species such as Entamoeba histolytica, Trypanosoma cruzi, Leshmania spp., Trichomonas vaginalis and Plasmodium spp. are actively proteolytic and contain multiple proteases that play a role in the life cycle, morphogenesis and infectivity (McKerrow et al. 1993). With respect to Giardia, despite evidence that proteolysis is involved in its nutrition, development and pathogenicity, relatively little is known. Initially, the intracelular activity of proteases in Giardia trophozoites was attributed to two major proteases corresponding to 105 and $40 \mathrm{kDa}$ bands detected by SDS-PAGE using gelatin as a substrate (Hare et al. 1989, Parenti 1991). Further investigations, however, have demonstrated the activity of multiple proteases in $G$. duodenalis trophozoites (Robertson et al. 1991, Williams \& Coombs 1995).

Most of these studies have investigated protease activity in trophozoites of the standard strains Portland 1 (P1) and WB, whereas few studies have been conducted

This research received financial support from Fapesp, grant no. 94/2946-6.

${ }^{+}$Corresponding author Fax: +55-14-6821.3744. E-mail: sgviana@ibb.unesp.br

Received 10 May 2002

Accepted 9 October 2002 on the protease activity in trophozoites of other axenic strains. Moreover, there is increasing interest to conduct studies in attempting to learn more about strains isolated and axenized in endemic areas, in order to know the biological, clinical and epidemiological inter-strain differences in these areas where the search for control measures is still frequent.

In the present investigation, we analyzed the protease activities in lysates of $G$. duodenalis trophozoites of two strains from a symptomatic (BTU-11) and an asymptomatic patient (BTU-10) isolated and axenized in Brazil, and the reference strain P1. The objectives were to analyse the protease activity in trophozoites and to determine if there are differences in such activity among the strains.

\section{MATERIALS AND METHODS}

Parasites and culture - Trophozoites of three axenic strains of G. duodenalis were studied. Two of them were isolated in Brazil, at the Giardiasis Laboratory (IB/Unesp) in Botucatu, São Paulo: BTU-10 from a 21 year-old asymptomatic male and BTU-11 from a 40 year-old symptomatic male presenting diarrhea, flatulence and abdominal cramps. Both strains were obtained from cysts in the patient's feces. The third strain P1, originally isolated by Meyer (1976) from a 36 year-old symptomatic female from Portland, Oregon (USA), was obtained from HW Pillai (Division of Geographic Medicine Tufts University, New England Medical Center, Boston, USA) and it was used as a standard strain. Trophozoites were grown in TYI-S-33 medium (Keister 1983 ) at $37^{\circ} \mathrm{C}$.

Preparation of G. duodenalis lysates - Extract of parasites were obtained according to Williams and Coombs (1995) procedures with slight modifications. Briefly, trophozoites were harvested at log-phase of growth (72-96 h) and after chiling in wet ice for $15 \mathrm{~min}$, washed (four times in $0.25 \mathrm{M}$ sucrose) by centrifugation $(1000 \mathrm{~g}, 20 \mathrm{~min}$, $4^{\circ} \mathrm{C}$ ) and the pellet stored at $-60^{\circ} \mathrm{C}$. The pellet of tropho- 
zoites $\left(2 \times 10^{8}\right.$ parasites $\left./ \mathrm{ml}\right)$ were then thawed at room temperature and the cells were disrupted at $4^{\circ} \mathrm{C}$ by four $10-15$ sec periods of ultrasonic treatment (Vibra cell ultrasonic processor VCX-400) with $60 \mathrm{sec}$ intervals. The extracts were centrifuged $\left(5000 \mathrm{~g}, 20 \mathrm{~min}, 4^{\circ} \mathrm{C}\right)$ and the supernatants aliquots stored at $-60^{\circ} \mathrm{C}$. Extracts to be employed in the analysis of protein profiles by SDS-PAGE and silver staining were treated with protease inhibitors [phenylmethylsulfonyl fluoride (PMSF, $1 \mathrm{mM}$ ), $N$-tosyl-L-phenylalanine chlorometyl ketone (TPCK, $1 \mathrm{mM}$ ), $N$ - $\alpha$-p-tosylL-lysine chlorometyl ketone (TLCK, $1 \mathrm{mM}$ ), iodacetamide (IAA, $1 \mathrm{mM}$ )]. The protein content was determined according to Bradford method (1976).

Electrophoresis (SDS-PAGE) - SDS-PAGE (Laemmli 1970), was developed using 7-14\% separating gels and $3 \%$ stacking gel. The samples were solubilized in sample buffer (1\% SDS, $128 \mathrm{mM} 2$-mercaptoethanol, $10 \%$ glicerol, $0.05 \%$ bromophenol blue, $500 \mathrm{mM}$ Tris- $\mathrm{HCl}, \mathrm{pH} 6.8$ ) and boiled for $3 \mathrm{~min}$; aliquots of $4 \mu \mathrm{g}$ of protein were applied to the gels. After electrophoresis, gels were stained with ammoniacal silver solution (Wray et al. 1981). Molecular weight markers were used.

Analysis of proteases by gelatin-SDS-PAGE - Preparations were mixed with sample buffer $(\mathrm{v} / \mathrm{v})$ and $5 \mu \mathrm{g}$ of proteins loaded without boiling on to polyacrylamide gels containing $0.2 \%$ copolymerized gelatin (Lockwood et al. 1987). Following electrophoresis, gels were washed in $2.5 \%$ Triton $\mathrm{X}-100\left(30 \mathrm{~min}, 37^{\circ} \mathrm{C}\right)$ to remove SDS and then $\left(15 \mathrm{~h}, 37^{\circ} \mathrm{C}\right.$ ) with $0.1 \mathrm{M}$ phosphate buffer ( $\mathrm{pH}$ 5.5) containing $1 \mathrm{mM}$ dithiothreitol (DTT). The bands of proteolysis were visualized by staining with $0.1 \%$ amido black in $30 \%$ methanol and $10 \%$ acetic acid and destaining in the same solution.

Effect of inhibitors - The effect of protease inhibitors (5 mM EDTA, 1 mM PMSF, 1 mME-64, 1 mM TPCK, 1 mM TLCK and $1 \mathrm{mM}$ IAA) was determined incubating the gels in $0.1 \mathrm{M}$ phosphate buffer ( $\mathrm{pH} 5.5$ ) containing $1 \mathrm{mM}$ DTT and the inhibitors $\left(15 \mathrm{~h}, 37^{\circ} \mathrm{C}\right)$.

Molecular masses determination - Molecular masses of fractionated proteins and bands of hydrolysis were determined by analysis in Image Master VDS (Pharmacia).

Reagents - Electrophoresis reagents, molecular weight markers, gelatin and inhibitors were purchase from Sigma Chemicals Co (St Louis, Mo, USA).

\section{RESULTS}

Protein profiles of lysates - To analyse the protein profiles, trophozoite lysates, treated and non-treated with protease inhibitors, were fractionated by SDS-PAGE and stained with silver (Fig. 1). Analysis of treated lysates revealed approximately 40 bands, with molecular masses ranging from 200 to $29 \mathrm{kDa}$ (Fig. 1). The protein patterns were similar and many bands were common to strains. The protein profiles of the non-treated lysates showed differences in the number of bands among the three strains (Fig 1). Most bands of BTU-10 lysate were conserved and the pattern was similar to that observed in the analysis of lysates treated with inhibitors. The protein profiles of non-treated lysates of BTU-11 and P1 strains revealed loss of higher molecular mass bands and increase in lower ones.

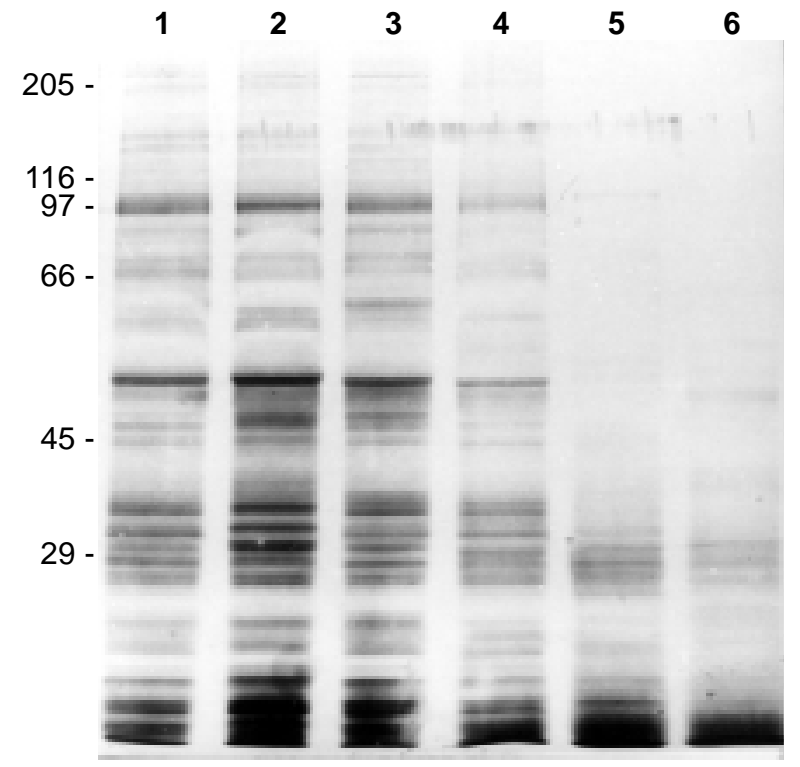

Fig. 1: analysis of SDS-PAGE (7-14\% gradient and silver stain) of proteins in Giardia duodenalis trophozoite lysates treated (lanes 1,2 and 3) and non-treated (lanes 4, 5 and 6) with protease inhibitors (1mM PMSF, $1 \mathrm{mM}$ TPCK, $1 \mathrm{mM}$ TLCK and $1 \mathrm{mM}$ IAA). The strains were loaded as follows: BTU-10 (lanes 1, 4), BTU-11 (lanes 2,5) and P1 (lanes 3,6). The protein loading for track was $4 \mu \mathrm{g}$. Molecular mass markers are shown on the left in kilodaltons (kDa).

Detection of proteases in trophozoite lysates by gelatin-SDS-PAGE - Protease activities in trophozoite lysates of $G$. duodenalis were investigated using polyacrylamide gels containing $0.2 \%$ copolymerized gelatin. Zones of hydrolysis were detected in trophozoite lysates of the Brazilian axenic strains and in the reference strain P1. However, the proteolysis band patterns demonstrated differences among strains (Figs 2a, 3a). The lysate of strain BTU-10, isolated from asymptomatic carrier, showed lower number of hydrolysis zones than the strains BTU-11 and $\mathrm{P} 1$, both isolated from symptomatic patients (Figs 2a, 3a). The pattern of protease activity in the BTU-10 lysate showed five bands: a group of three bands with molecular masses of 92,86 and 74 $\mathrm{kDa}$ (bands 1-3) and two bands of 54 and $50 \mathrm{kDa}$. The pattern of BTU-11 and P-strains revealed 10-11 bands of hydrolysis with molecular masses ranging from 92 to $23 \mathrm{kDa}$ and 116 to $23 \mathrm{kDa}$, respectively. The BTU-11 lysate revealed hydrolysis bands ranging from 92,86 and $72 \mathrm{kDa}$, two bands of 66 and $64 \mathrm{kDa}$, a prominent band of $50 \mathrm{kDa}$ and a group of diffuse bands with molecular masses ranging from 32 to $23 \mathrm{kDa}$. Analysis of protease activities in P1 lysate showed a band of $116 \mathrm{kDa}$, a group of three bands of 92,86 and $74 \mathrm{kDa}$, a faint band of $66 \mathrm{kDa}$, two bands of 62 and $58 \mathrm{kDa}$, a prominent band of $50 \mathrm{kDa}$ and a group of light bands with molecular masses ranging from 32 to $23 \mathrm{kDa}$. The $50 \mathrm{kDa}$ band was the most pronounced zone of hydrolysis observed mainly in the lysates of strains BTU-11 and P1.

Effect of protease inhibitors - The results of the effect of PMSF, TLCK and E-64 on protease activities are given in Fig 2 . The activity of all proteases in lysates was inhib- 
ited with addition of E-64 (Fig. 2d) whereas PMSF did not detectably inhibited hydrolysis (Fig. 2b). The inclusion of TLCK resulted in inhibition of the great part of hydrolysis bands (Fig. 2c). Fig. 3 shows the effect of iodoacetamide, EDTA and TPCK. The addition of iodoacetamide resulted in marked reduction of all proteases activities (Fig. 3b). Incubation of gels containing resolved proteases in buffer containing EDTA (Fig. 3c) had no effect on the proteolytic activity of the three lysates studied. The addition of TPCK resulted in inhibition of many hydrolysis bands (Fig. 3d). The incubation of gels with TLCK and TPCK had no effect on the proteases corresponding to the zones of hydrolysis ranging from 92-72 kDa (Figs 2c, 3d).

\section{DISCUSSION}

In the present study, we have demonstrated the proteolytic activity in $G$. duodenalis trophozoites of strains isolated in Brazil, as well as some inter-strain differences among the observed band patterns.

In contrast to early investigations that detected the activity of only two major proteases in $G$. duodenalis
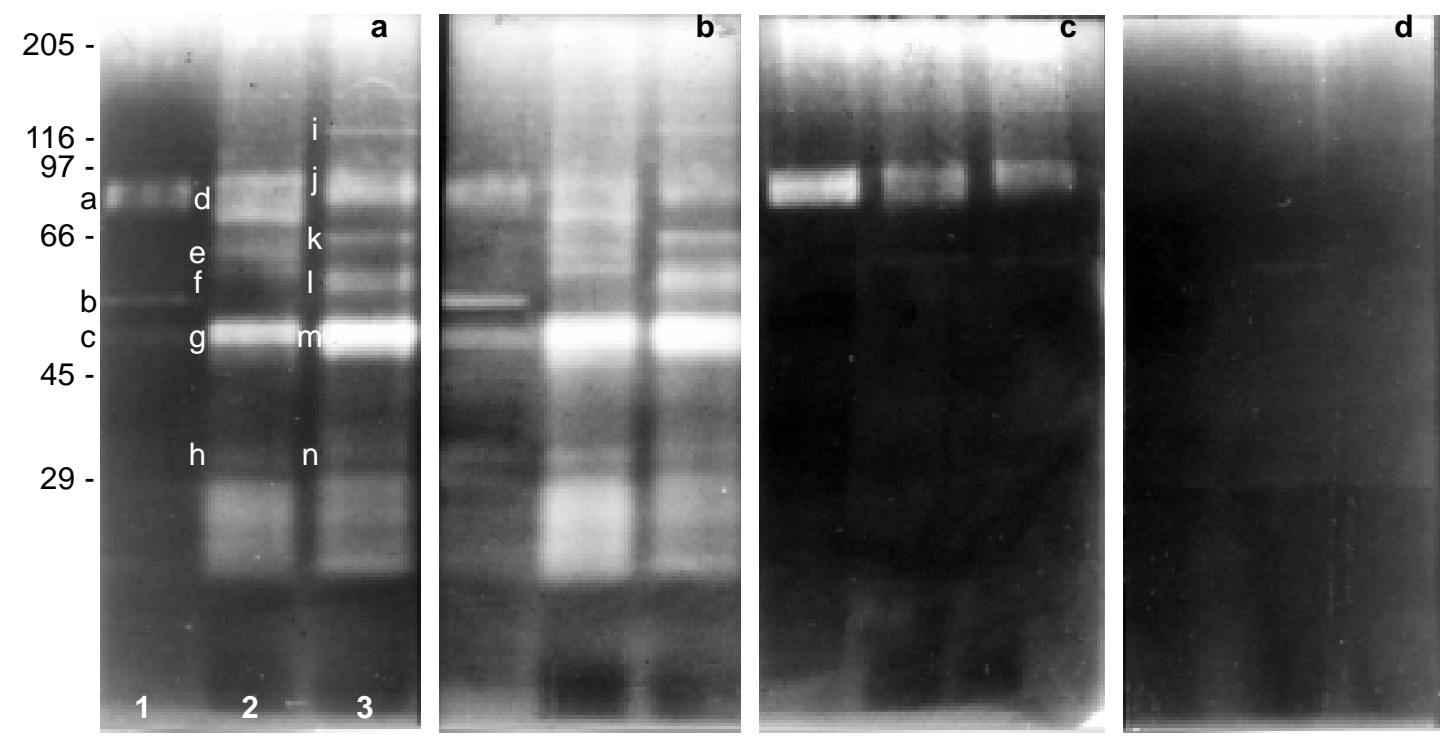

Fig. 2: effect of inhibitors on the protease activities in Giardia duodenalis trophozoite lysates of BTU-10 (lane 1), BTU-11 (lane 2) and $\mathrm{P} 1$ (lane 3). Gels were incubated for $15 \mathrm{~h}$ at $37^{\circ} \mathrm{C}$ in phosphate buffer pH 5.5 with $1 \mathrm{mM}$ DTT (a) and with $1 \mathrm{mM}$ DTT and inhibitor (b, c, d). Lysates in the presence of PMSF (b), TLCK (c), and E-64 (d). The protein loading for track was $5 \mu$ g. Molecular mass mark ers are shown on the left in kilodaltons $(\mathrm{kDa}$ ). The letters on the left of lanes 1, 2 and 3 designate regions of hydrolysis as follows: (a, j) 92-78 kDa; (b) $54 \mathrm{kDa}$; (c, g, m) $50 \mathrm{kDa}$; (d) $92-72 \mathrm{kDa}$; (e, k) $66 \mathrm{kDa}$; (f) $64 \mathrm{kDa}$; (h, n) 32-23 kDa; (i) $116 \mathrm{kDa}$; (1) $62-58 \mathrm{kDa}$.
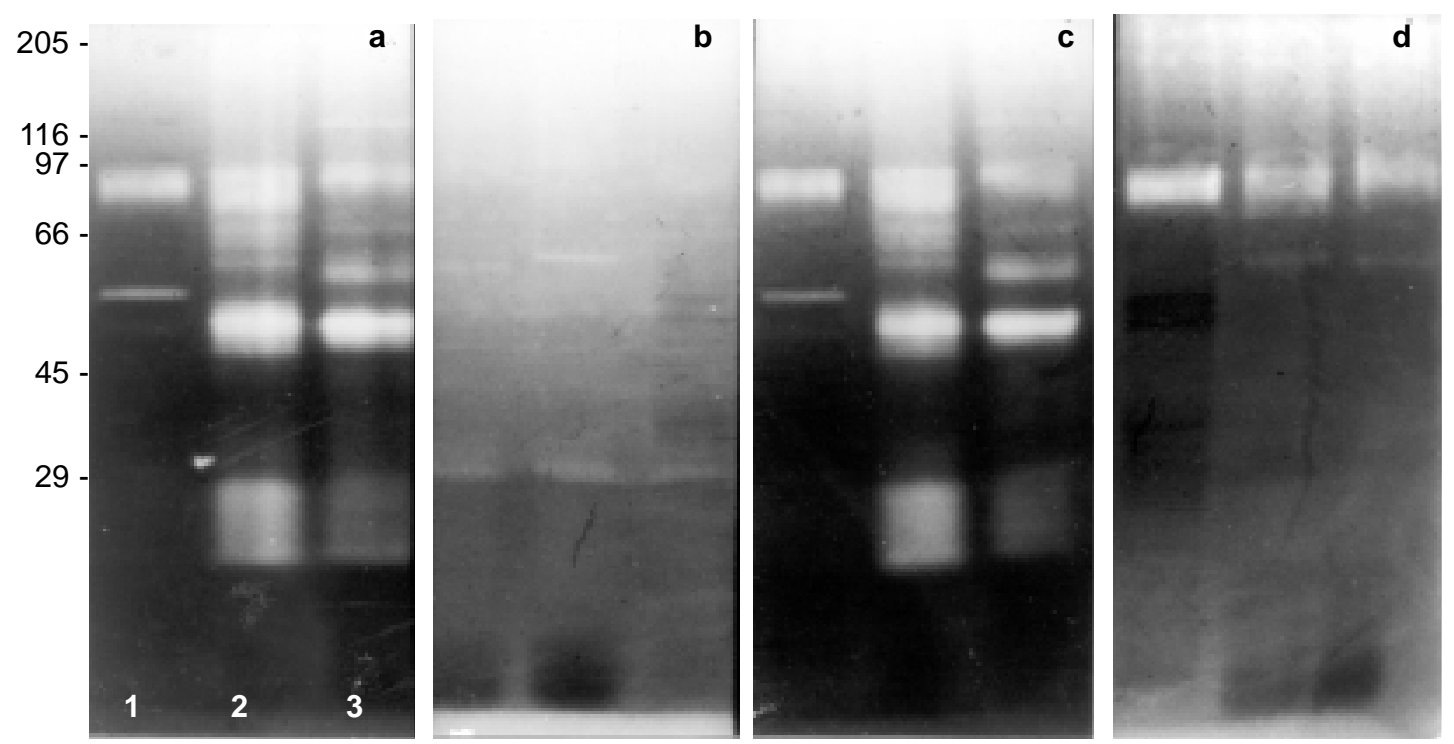

Fig. 3: effect of inhibitors on the protease activities in Giardia duodenalis trophozoite lysates of BTU-10 (lane 1), BTU-11 (lane 2) and P1 (lane 3). Gels were incubated as described in the Fig. 2 with $1 \mathrm{mM}$ DTT (a) and with $1 \mathrm{mM}$ DTT and inhibitor (b, c, d). Lysates in the presence of iodoacetamide (b), EDTA (c) and TPCK (d). The protein loading for track was $5 \mu \mathrm{g}$. Molecular mass markers are shown on the left in kilodaltons $(\mathrm{kDa})$. 
trophozoites (Hare et al. 1989, Parenti 1991), recent studies have demonstrated multiple proteases in the parasite (Robertson et al. 1991, Williams \& Coombs 1995, Jimenez et al. 2000). Our results corroborate latter findings, since we detected five bands in the trophozoite lysate of BTU10 strain, and 10-11 bands in the lysates of BTU-11 and P1 strains. Some of the main proteases detected showed molecular masses similar to those demonstrated previously. In the analysis of strain P1, Williams and Coombs (1995) detected as the proteases producing the most pronounced hydrolysis zones those with molecular masses of $120 \mathrm{kDa}, 80 \mathrm{kDa}, 73 \mathrm{kDa}, 66 \mathrm{kDa}, 57 \mathrm{kDa}, 33 \mathrm{kDa}$ and 31 $\mathrm{kDa}$. More recently, Jimenez et al. (2000) identified proteolytic bands at $16,20,66,82,108$ and $120 \mathrm{kDa}$ in the lysate of P1 strain. Employing the same strain, we distinguished the bands with molecular masses of $116 \mathrm{kDa}, 92-$ $78 \mathrm{kDa}$ (bands 2-4), $64 \mathrm{kDa}, 58 \mathrm{kDa}, 50 \mathrm{kDa}$ and a group of diffuse bands in the region of 32-23 kDa. The Brazilian strains lysates revealed bands of hydrolysis with similar molecular masses. Another important finding was the detection in all strains of a band with an approximate molecular mass of $50 \mathrm{kDa}$. Moreover, this band was the most pronounced zone of hydrolysis in the profiles of strains BTU-11 and P1, isolated from symptomatic cases. We may wonder whether this protease is an important component in the strains obtained from symptomatic patients. To confirm this possibility, the purification and characterization of the enzyme may be warranted.

In agreement with previous reports (Hare et al. 1989, Parenti 1991, Robertson et al. 1991, Werries et al. 1991, Williams \& Coombs 1995, Jimenez et al. 2000), our results showed that cysteine proteases predominate in trophozoite lysates of G. duodenalis strains, including the Brazilian ones. The addition of cysteine protease inhibitors E-64 and iodoacetamide (IAA) resulted in a striking inhibition of all protease activities. It is important to emphasize that E-64 is a specific and an effective irreversible inhibitor of cysteine proteases. No effect was observed when lysates were incubated with the inhibitors PMSF and EDTA, which act on serine proteases and metalloproteases, respectively. TLCK and TPCK affected the activity of great part of proteases, except the proteases with molecular masses of 92-72 kDa. TLCK and TPCK are inhibitors of some trypsin-like and chymotripsin-like serine proteases, respectively, but they can affect some cysteine proteases. This can explain why these inhibitors affected the activity of great part of proteases of the three lysates studied. The effect of these inhibitors on $G$. duodenalis cysteine proteases was observed in previous studies (Hare et al. 1989, Parenti 1991, Williams \& Coombs 1995). So, the lysates employed in our study contain multiple proteases and the cysteine proteases predominate in trophozoite of G. duodenalis. The cysteine proteinases are a group of enzymes that appears to be most abundant in parasitic protozoa (North et al. 1990). Nowadays, isolation and sequence analysis of genes encoding cysteine proteinases is a relevant area of investigation. It will be interesting to see whether the multiple cysteine proteinases have different functions. The genes encoding different Giardia enzymes have been characterized (Hiltpold et al. 1999, Bunjun et al. 2000, Dan \& Wang 2000). In relation to cysteine proteinases, structural and kinetic analyses of these enzymes should provide important insights on their biochemical function and may be helpful to design chemotherapeutic agents for the treatment of giardiasis based on inhibitors or substrates of these enzymes.

An interesting finding of our study was the differences among the hydrolysis band patterns of the strains BTU-10, BTU-11 and the reference strain P1. The strain BTU-10 obtained from asymptomatic patient showed fewer hydrolysis zones than the strains BTU-11 and P1, both isolated from symptomatic patients. The profiles of strains BTU-11 and P1 revealed 10-11 zones of hydrolysis with molecular masses ranging from 92 to $23 \mathrm{kDa}$ and 116 to 23 $\mathrm{kDa}$, respectively. The previous SDS-PAGE analysis of protein profiles of lysates non-treated with protease inhibitors provides support for this finding, for not only proteolysis was evident in all lysates but there were also differences in the number of protein bands. The great part of protein bands disappeared in the profiles of BTU-11 and P1 strains. The detection of loss of higher molecular masses bands and increase in lower ones is a strong indicative of proteolysis in the protein samples. In contrast, the analysis revealed the maintenance of the protein bands in the BTU-10 non-treated lysate.

Despite of the reduced number of strains analysed, we have observed that the strains isolated from symptomatic patients revealed more proteolytic bands than the strain isolated from the asymptomatic patient. Strain differences related to proteolytic activity in Giardia trophozoites are still scarce, since most studies have investigated protease activities mainly in trophozoites of the standard strains P1 and WB, without comparison among other strains.

This finding may one wonder whether proteases may be associated with virulence and pathogenicity of strains of G. duodenalis. Studies with the intestinal protozoa Entamoeba histolytica have correlated virulence and pathogenicity with the amount of crude proteolytic activity in amebic secretions and in lysates (Keene et al. 1986, 1990, Que et al. 1997). In relation to G. duodenalis, further studies with a greater number of strains isolated from symptomatic and asymptomatic patients are needed before this correlation can be established. Even so, our results hold the perspective for the understanding about the protease activity in $G$. duodenalis trophozoites of axenic strains isolated in an endemic area.

\section{REFERENCES}

Bunjun S, Stathopoulos C, Graham D, Min B, Kitabatake M, Wang AL, Wang CC, Vivares CP, Weiss L, Soll D 2000. A dual-specificity aminoacyl-tRNA synthetase in deep-rooted eukaryote Giardia lamblia. Proc Natl Acad Sci USA 97: 12997-13002.

Bradford MM 1976. A rapid and sensitive method for the quantification of microgram quantities of protein utilizing the principle of protein-dye bindings. Anal Biochem 72: 248254.

Dan M, Wang CC 2000. Role of alcohol dehydrogenase E (ADHE) in the energy metabolism of Giardia lamblia. Mol Biochem Parasitol 109: 271-273.

Hare DF, Jarrol EL, Lindmark DG 1989. Giardia lamblia: characterization of proteinase activity in trophozoites. Exp Parasitol 68: 168-175. 
Hiltpold A, Thomas RM, Kohler P 1999. Purification and characterization of recombinant pyruvate phosphate dikinase from Giardia. Mol Biochem Parasitol 104: 157-169.

Jimenez JC, Uzcanga G, Zambrano A, Di Prisco MC, Lynch NR 2000. Identification and partial characterization of excretory/secretory products with proteolytic activity in Giardia intestinalis. J Parasitol 86: 859-862.

Keene WE, Petitt MG, Allen S, McKerrow JH 1986. The major neutral proteinase of Entamoeba histolytica. J Exp Med 163: 536-549.

Keene WE, Hidalgo ME, Orozco E, McKerrow JH 1990. Entamoeba histolytica: correlation of the cytopathic effect of virulent trophozoites with secretion of a cysteine proteinase. Exp Parasitol 71: 199-206.

Keister DB 1983. Axenic culture of Giardia lamblia in TYI-S33 medium supplemented with bile. Trans $R$ Soc Trop Med Hyg 77: 487-488.

Laemmli UK 1970. Cleavage of structural protein during the assembly of the bacteriophage T4. Nature 277: 680-685.

Lockwood B, Scott KI, Bremner AF, Coombs GH 1987. The use of a highly sensitive electrophoretic method to compare the proteinases of trichomonads. Mol Biochem Parasitol 24: 89-95.

McKerrow JH, Sun E, Rosenthal J, Bouvier J 1993. The pro- teases and pathogenicity of parasitic protozoa. Ann Rev Microbiol 47: 821-853.

Meyer EA 1976. Giardia lamblia: isolation and axenic cultivation. Exp Parasitol 39: 101-105.

North MJ, Mottram JC, Coombs GH 1990. Cysteine proteinases of parasitic protozoa. Parasitol Today 6: 270-275.

Parenti DM 1991. Characterization of a thiol proteinase in Giardia lamblia. J Infect Dis 160: 1076-1080.

Que X, Reed SL 1997. The role of extracellular cysteine proteinases in pathogenesis of Entamoeba histolytica. Parasitol Today 13: 190-194.

Robertson CD, Irvine JW, Brown K, Jooma L, Seely C, Young CJ, Coombs GH 1991. Proteinases of Giardia lamblia trophozoites. Trans R Soc Trop Med Hyg 85: 844.

Werries E, Franz A, Hippe H, Acyl Y 1991. Purification and substrate specificity of two proteinases of Giardia lamblia trophozoites. J Parasitol 38: 378-383.

Williams AG, Coombs GH 1995. Multiple protease activities in Giardia intestinalis trophozoites. Int J Parasitol 25: 771778.

Wray W, Boulikas T, Wray VP, Kancol JR 1981. Silver staining of proteins in polyacrylamide gels. Anal Biochem 118: 197203. 